CERN-TH.7375/94

August 1994

\title{
An Improved Estimator for the Correlation Function of 2D Nonlinear Sigma Models
}

\author{
Martin Hasenbusch \\ CERN, Theory Division, \\ CH-1211 Genève 23, Switzerland ${ }^{1}$
}

\begin{abstract}
I present a new improved estimator for the correlation function of 2D nonlinear sigma models. Numerical tests for the 2D $X Y$ model and the 2D $O(3)$-invariant vector model were performed. For small physical volume, i.e. a lattice size small compared to the bulk correlation length, a reduction of the statistical error of the finite system correlation length by a factor of up to 30 compared to the cluster-improved estimator was observed. This improvement allows for a very accurate determination of the running coupling proposed by $\mathrm{M}$. Lüscher et al. for 2D $O(N)$ invariant vector models.
\end{abstract}

CERN-TH.7375/94

August 1994

${ }^{1}$ Address after September 30, 1994: DAMTP, Silver Street, Cambridge, CB3 9EW, England 


\section{Introduction}

Almost twenty years ago Nightingale [1] pointed out that the correlation length of a finite system can be used to trace the renormalization group flow of a statistical model. He demonstrated the validity of his approach at the example of the 2D Ising model using its exact solution [2].

Lüscher computed the mass gap (the inverse correlation length) for the 2D $O(3)$ vector model on finite lattices using perturbation theory [3]. In ref. [4] Lüscher, Weisz and Wolff used the mass gap of finite systems to define a running coupling for $2 \mathrm{D}$ asymptotically free models. The aim of their study was to match the perturbative, i.e. small distance, regime with the nonperturbative large distance regime, using $\mathrm{MC}$ simulations for large distances and in the cross-over region. The high numerical accuracy that they reached for the step scaling function of this particular running coupling of the $2 \mathrm{D} O(3)$-invariant vector model was based on the use of the efficient cluster algorithm [5] combined with a variance reducing cluster-improved estimator [6]. However one has to note that up to now this powerful simulation technique only applies to a rather restricted class of models. I will discuss an alternative improved estimator that does not rely on the cluster algorithm and hence can be applied to a larger class of symmetry groups.

The concept of improved estimators is rather general and by no means restricted to the cluster algorithm. The basic idea of improved estimators is to integrate analytically or by standard numerical methods those degrees of freedom that are most important for the fluctuations of the observable in question. For local fluctuations this can be achieved easily. Parisi et al. [7] proposed to improve the estimator of the Wilson-loop correlation-function by integrating over the link-variables on the Wilson-loops.

I will show that such ideas also apply to large range correlation functions of certain models. The estimator is based on an embedding of a one-dimensional model. The degrees of freedom of this embedded model are collective transformations of all spins in a given time-slice.

This paper is organized as follows. In section 2 I define the new improved estimator for two-dimensional $O(N)$ invariant vector models. In section 3 I summarize theoretical predictions for the running coupling of 2D $O(N)$-invariant vector models. In section 4 I present numerical results for the 2D $O(2)$-invariant vector $(X Y)$ model and the 2D $O(3)$-invariant vector model. The conclusions are given in section 5 . 


\section{The improved estimator}

First let us discuss the strategy applied for finding an improved estimator for an observable $A$ of a statistical model with field variables $\phi$. The expectation value of $A$ is given by

$$
\langle A\rangle=\frac{\int D \phi \exp (-S(\phi)) A(\phi)}{\int D \phi \exp (-S(\phi))} .
$$

We split the set of field variables into two parts $\phi=(\bar{\phi}, \Phi)$, where $\Phi$ is chosen such that the integrals over $\Phi$ can be performed exactly for any fixed $\bar{\phi}$. Let us define the improved estimator by

$$
A_{\text {imp }}(\phi)=A_{\text {imp }}(\bar{\phi})=\langle A\rangle_{\text {cond }}=\frac{\int D \Phi \exp (-S(\bar{\phi}, \Phi)) A(\bar{\phi}, \Phi)}{\int D \Phi \exp (-S(\bar{\phi}, \Phi))},
$$

where $\langle\ldots\rangle_{\text {cond }}$ denotes the conditional, i.e. $\bar{\phi}$ dependent expectation value. It is easy to check that $\left\langle A_{i m p}\right\rangle=\langle A\rangle$ and also that the variance of the improved estimator $A_{\text {imp }}$ is reduced compared to that of $A$

$$
\left\langle A^{2}\right\rangle-\langle A\rangle^{2} \geq\left\langle A_{i m p}^{2}\right\rangle-\left\langle A_{i m p}\right\rangle^{2}
$$

Of course, the remaining non-trivial question is to identify, for a given model and observable, the $\Phi$ such that the integration over $\Phi$ can be performed with reasonable effort and leads to a considerable reduction of the variance.

Now let me explain the improved correlation function estimator for the $O(N)$ invariant vector models. The action is given by

$$
S=-\beta \sum_{x, t} \sum_{\alpha=1}^{N}\left(s_{\alpha}(x, t) s_{\alpha}(x+1, t)+s_{\alpha}(x, t) s_{\alpha}(x, t+1)\right)
$$

where $s_{\alpha}(x, t)$ are the components of a real $N$-component unit vector. The lattice has extension $T$ in the time $(t-)$ direction and extension $L$ in spatial $(x-)$ direction. For simplicity we set the lattice-spacing $a$ equal to 1 in this section. In time direction we employ open boundary conditions while in $x$-direction periodic boundary conditions are employed, i.e. identify $x=L+1$ with $x=1$ and $x=0$ with $x=L$. We choose left-multiplications of all spins in a time-slice $t$ with the same $O(N)$-matrix $R(t)$ as degrees of freedom of the $1 \mathrm{D}$ embedded model. The improved estimator is then constructed by integrating over the $R(t)$ exactly. The conditional, i.e. $s_{\alpha}$ dependent, action of the embedded models is given by

$$
S_{\text {cond }}(R)=-\beta \sum_{x, t} \sum_{\alpha=1}^{N}\left(\sum_{\delta=1}^{N} R_{\alpha, \delta}(t) s_{\delta}(x, t)\right)\left(\sum_{\gamma=1}^{N} R_{\alpha, \gamma}(t+1) s_{\gamma}(x, t+1)\right)
$$


Note that contributions from spatial bonds stay constant under the transformation, and can hence be ignored. The conditional partition function is given by

$$
Z_{\text {cond }}=\int D R \exp \left(-S_{\text {cond }}(R)\right)
$$

where $D R=\prod_{t} d R(t)$ and $d R$ denotes the Haar measure of the $O(N)$ group. Next let us introduce relative rotations $X(t, t+1)$ of two neighbouring time-slices by

$$
X(t, t+1)=R^{-1}(t) R(t+1)
$$

All $R(t)$ can be expressed in terms of $R(1)$ and the $X(t, t+1)$.

$$
R(t)=R(1) X(1,2) \ldots X(t-2, t-1) X(t-1, t)
$$

Now it becomes clear why free boundary conditions in time direction are used. Periodic boundary conditions in time direction would require that the product of all $X(t, t+1)$ on the lattice is equal to the unit matrix. In terms of the $X$ the action of the conditional model is given by

$$
S_{\text {cond }}(X)=-\sum_{t} \sum_{\alpha=1}^{N} \sum_{\gamma=1}^{N} X_{\alpha, \gamma}(t, t+1) Q_{\alpha, \gamma}(t, t+1)
$$

where $Q$ is given by

$$
Q_{\alpha, \gamma}(t, t+1)=\beta \sum_{x} s_{\alpha}(x, t) s_{\gamma}(x, t+1) .
$$

The properties of the Haar measure allow also to rewrite the measure in terms of the relative rotations $X$

$$
Z_{\text {cond }}=\int d R(1) \int D X \exp \left(-S_{\text {cond }}(X)\right)
$$

where the integration over $R(1)$ just gives a constant factor that hence can be ignored. The conditional partition function factorizes in two-slice partition functions

$$
Z_{\text {cond }}=\prod_{t=1}^{t=T-1} z(t, t+1)
$$

with

$$
z(t, t+1)=\int d X \exp \left(-\sum_{\alpha=1}^{N} \sum_{\gamma=1}^{N} X_{\alpha, \gamma}(t, t+1) Q_{\alpha, \gamma}(t, t+1)\right)
$$


where $d X$ denotes the Haar measure of the $O(N)$ group.

Next let us turn to the measurement of time-slice correlation functions. The time-slice correlation function is defined by

$$
G(\tau)=\sum_{\alpha} S_{\alpha}(t) S_{\alpha}(t+\tau)
$$

where

$$
S_{\alpha}(t)=\sum_{x} s_{\alpha}(x, t) .
$$

The improved estimator of the time-slice correlation function is given by

$$
G_{i m p}(\tau)=\langle G(\tau)\rangle_{\text {cond }}=\sum_{\alpha}\left\langle\sum_{\delta} R_{\alpha, \delta}(t) S_{\delta}(t) \sum_{\gamma} R_{\alpha, \gamma}(t+\tau) S_{\gamma}(t+\tau)\right\rangle_{\text {cond }},
$$

where

$$
\langle A(R)\rangle_{\text {cond }}=\frac{\int D R \exp \left(-S_{\text {cond }}(R)\right) A(R)}{Z_{\text {cond }}}
$$

is the conditional expectation value of an observable $A(R)$. Again we can re-express things in terms of relative rotations

$$
\begin{aligned}
G_{i m p}(\tau) & =\sum_{\alpha} \sum_{\gamma}\left\langle S_{\alpha}(t) X_{\alpha, \gamma}(t, t+\tau) S_{\gamma}(t+\tau)\right\rangle_{\text {cond }} \\
& =\sum_{\alpha} \sum_{\gamma} S_{\alpha}(t)\left\langle X_{\alpha, \gamma}(t, t+\tau)\right\rangle_{\text {cond }} S_{\gamma}(t+\tau),
\end{aligned}
$$

where $X(t, t+\tau)=R^{-1}(t) R(t+\tau)$. Using the laws of matrix calculus and the factorization of the conditional partition function we obtain

$$
\langle X(t, t+\tau)\rangle_{c o n d}=\langle X(t, t+1)\rangle_{c o n d} \ldots\langle X(t+\tau-1, t+\tau)\rangle_{c o n d} .
$$

Hence the evaluation of the conditional expectation values is reduced to the evaluation of the low dimensional integral

$$
\langle X(t, t+1)\rangle_{\text {cond }}=\frac{\int d X \exp \left(\sum_{\alpha, \gamma} X_{\alpha, \gamma} Q_{\alpha, \gamma}(t, t+1)\right) X}{\int d X \exp \left(\sum_{\alpha, \gamma} X_{\alpha, \gamma} Q_{\alpha, \gamma}(t, t+1)\right)}
$$

\subsection{Performing the integrals for $\mathrm{N}=2$ and 3 .}

For $N=2$ this integral can be solved in closed form. One obtains

$$
X_{11}=X_{22}=\frac{I_{1}(\kappa)}{I_{0}(\kappa)} \frac{Q_{11}+Q_{22}}{\kappa}
$$


and

$$
X_{21}=-X_{12}=\frac{I_{1}(\kappa)}{I_{0}(\kappa)} \frac{Q_{21}-Q_{12}}{\kappa},
$$

where

$$
\kappa=\sqrt{\left(Q_{11}+Q_{22}\right)^{2}+\left(Q_{21}-Q_{12}\right)^{2}}
$$

and $I_{0}$ and $I_{1}$ are modified Bessel-functions.

For $N=3$ I could not find an exact solution for the integrals. For practical reasons I restricted the integration to the $S O(3)$ subgroup of $O(3)$.

Using the property

$$
\int d X f(X)=\int d X f(U X V)
$$

of the Haar measure, where $U$ and $V$ are elements of the group manifold, the integrand

$$
\int d X \exp \left(\operatorname{Tr} X Q^{T}\right)=\int d X \exp \left(\operatorname{Tr} U X V Q^{T}\right)=\int d X \exp \left(\operatorname{Tr} X V Q^{T} U\right)
$$

can be transformed such that the source $\tilde{Q}=V Q^{T} U$ becomes diagonal.

Now I parametrized the $S O(3)$ using the Euler-angles. One of the 3 integrations can be performed analytically. The remaining 2D integral I solved numerically.

If all three sums $q_{1}=\tilde{Q}_{11}+\tilde{Q}_{22}, q_{2}=\tilde{Q}_{11}+\tilde{Q}_{33}$ and $q_{3}=\tilde{Q}_{22}+\tilde{Q}_{33}$ are positive the integrand takes its maximal value for $X$ equals the unit matrix. I performed a saddle-point approximation around this maximum up to order $q^{-8}$. Comparing with the numerical solution of the integral I checked that for $\min \left(q_{1}, q_{2}, q_{3}\right)>13$ the difference between the truncated series and the exact result for the components of $\langle X\rangle_{\text {cond }}$ is smaller than $5 \times 10^{-8}$.

In the remaining cases I employed the numerical integration described above.

\section{Theoretical Predictions}

\subsection{The 2D $O(N)$-invariant vector model with $N>2$}

The 2D $O(N)$-invariant vector models can formally be defined by the continuum action

$$
S=-\frac{1}{2 g^{2}} \int d^{2} x \partial_{\mu} s(x) \partial_{\mu} s(x)
$$

where $s$ is a real $N$-component unit vector and $g^{2}$ is the coupling constant. The corresponding Wilson lattice action I have already given in eq. (雨). The 2D $O(N)$ invariant vector models with $N>2$ are believed to be asymptotically free [12]. This 
means that the model has a finite correlation length for all finite $\beta$ and the coupling $g^{2}$ vanishes with decreasing length scale. The running coupling

$$
\bar{g}^{2}=\frac{2 L}{(N-1) \xi(L)}
$$

introduced in ref. [4] is chosen such that $g^{2}$ and $\bar{g}^{2}$ coincide at 1-loop order of perturbation theory. The flow of $\bar{g}^{2}$ is given by the Callan-Symanzik $\beta$-function

$$
\beta\left(\bar{g}^{2}\right)=-L \frac{\partial \bar{g}^{2}}{\partial L}
$$

where $\beta(u)$ is given for small $u$ by

$$
\beta(u)=-u^{2} \sum_{l=0}^{\infty} b_{l} u^{l}
$$

The first three coefficients of this expansion are know from perturbation theory [4]

$$
\begin{aligned}
& b_{0}=(N-2) /(2 \pi) \\
& b_{1}=(N-2) /(2 \pi)^{2} \\
& b_{2}=(N-1)(N-2) /(2 \pi)^{3} .
\end{aligned}
$$

Of course on the lattice only finite rescalings are allowed. Therefore in ref. [4] a continuum step-scaling function $\sigma(s, u)$ is introduced, where $s=L^{\prime} / L$ is the scaling factor. In addition the step-scaling function on the lattice $\Sigma(s, u, a / L)$ is affected by finite lattice-spacing artefacts. The continuum step scaling function is recovered in the limit of vanishing $a$.

$$
\lim _{a \rightarrow 0} \Sigma(s, u, a / L)=\sigma(s, u)
$$

From the analysis of the cutoff dependence of Feynman diagrams [8] one knows [4] that up to logarithmic corrections the leading finite lattice-spacing artefacts are $O\left(a^{2}\right)$. The dependence of the relative error

$$
\delta(u, a / L)=\frac{\Sigma(2, u, a / L)-\sigma(2, u)}{\sigma(2, u)}=\delta_{1}(u, a / L) u+\delta_{2}(u, a / L) u^{2} \ldots
$$

can be computed in perturbation theory. Some of the results given in ref. [4] for $N=3$ are summarized in table 2 . 


\subsection{The 2D $X Y$ model}

In contrast to the 2D $O(N)$-invariant vector models with $N>2$ the 2D $X Y$ model undergoes a phase transition at a finite $\beta$. The most accurate value for the critical point is $\beta_{c}=1.1197(5)$ [11]. The phase transition of the 2D $X Y$ model is described by the Kosterlitz-Thouless (KT) theory [10].

For $\beta<\beta_{c}$ the correlation length $\xi_{b u l k}$ is finite and as $\beta \rightarrow \beta_{c}$ it diverges like

$$
\xi_{\text {bulk }} \propto \exp \left(C\left(\beta_{c}-\beta\right)^{-1 / 2}\right)
$$

where $C$ and $\beta_{c}$ are model dependent. The finite $\xi_{b u l k}$ implies that $\bar{g}^{2}$ increases linearly with $L$ in the limit of large $L$.

For $\beta>\beta_{c}$ the theory is massless, i.e., the correlation function decays algebraically in the infinite volume. The large distance behaviour in the massless phase is described by an effective Gaussian theory

$$
S_{\text {eff }}=\frac{1}{2} \beta_{\text {eff }} \int d^{2} x(\nabla \phi(x))^{2},
$$

where $\phi$ is the angle of the spin. The critical point is characterized by $\beta_{\text {eff }}=2 / \pi$. For the Gaussian action one obtains $\bar{g}^{2}=1 / \beta_{\text {eff }}$ independent of $L$.

The improvement of course depends on the distance $\tau$ that is considered. In general the statistical error will increase exponentially with $\tau$. As an example I studied the correlation function $G(\tau)$ and the effective correlation length $\xi_{\text {eff }}(\tau)$ at $\beta=1.8932$ and $L=64$ in more detail.

In figure 1 the relative statistical error of the correlation function is plotted as a function of the distance $\tau$ for the three estimators. The error of the new improved estimator is smaller than that of the other estimators for all distances considered and stays almost constant in the studied region. In figure 2 the relative statistical error of $\xi_{e f f}(\tau)$ is plotted. As for the correlation function itself the error of the improved estimator is strictly smaller than that of the other two estimators and stays almost constant with increasing $\tau$, while the error of the other two estimators rapidly increases with increasing distance $\tau$.

Next I tried to reproduce two of the results for the step-scaling function

$$
\sigma(2,0.7383)=0.8166(9)
$$

and

$$
\sigma(2,1.0595)=1.2641(20)
$$

given in ref. [4]. Therefore I determined $\Sigma(2,0.7383, a / L)$ and $\Sigma(2,1.0595, a / L)$ for various $L / a$ in the range $L / a=6$ up to $L / a=32$. First one has to find for each 
$L / a$ the $\beta$ at which $\bar{g}^{2}=0.7383$ or $\bar{g}^{2}=1.0595$ is reached. For $L / a \leq 16$ I took the $\beta$ 's found in ref. [4] as first guess. In order to correct the small deviations from the wanted value of $\bar{g}^{2}$ I determined the slope of $\bar{g}^{2}(\beta)$ from runs with smaller statistics (10000 measurements) at $\beta$-values slightly smaller and larger. For the $L$ not studied in ref. [4] I started with a guess obtained from extrapolating the data for smaller $L$. Then I computed a second guess from two simulations with 10000 measurements in the neighbourhood of the first guess. The result of the simulation with 100000 measurements at this second guess for $\beta$ I give in table 1 . The final correction is then done using the result for the slope of $\bar{g}^{2}(\beta)$.

Next I simulated on a lattice of size $L^{\prime} / a=s L / a$ with $s=2$ at the $\beta$-values obtained above. The results of these simulations are also given in table 1 .

The resulting $\bar{g}^{2}$ gives the finite lattice-spacing approximation $\Sigma(s, u, L / a)$ of $\sigma(s, u)$. In order to obtain the continuum result the limit $a \rightarrow \infty$ has to be taken. For $\Sigma(s, 1.0595, L / a)$ the finite lattice-spacing artefacts even have a different sign than predicted by perturbation theory [4]. Nevertheless I assume, following ref. [4] that the finite lattice spacing artefacts vanish approximately like $(a / L)^{2}$. Hence one might improve the convergence towards $\sigma(s, u)$ with decreasing $a / L$ by fitting the data for $\Sigma(s, u, L / a)$ according to an ansatz

$$
\Sigma(2, u, a / L)=\sigma(2, u)+c_{2}(a / L)^{2}+c_{4}(a / L)^{4} \ldots
$$

Results for such fits are given in table 3. The statistical error of the $\beta$ 's is taken into account in the statistical error of $\Sigma(2, u, a / L)$. Even when including all data points the $\chi^{2} /$ dof is small. The result for $\sigma(2,1.0595)$ stays stable when data-points with small $L$ are discarded; also $c_{2}$ stays stable. My final estimate $\sigma(2,1.0595)=$ $1.2639(10)$, taken from the fit where the two smallest $L / a$-values are discarded, is consistent with that of ref. [4], while the statistical error is reduced by more than a factor of 2. Including larger lattice sizes also reduces the danger of systematical errors. A similar analysis leads to $\sigma(2,0.7383)=0.8156(4)$ which is again consistent with ref. [4.

Next I simulated the model at $\beta=5.0$ on lattices of size $L=6,8,12,16,24,32$ and 48. The statistical error of the correlation length determined from the conventional and the cluster estimator were approximately the same. But with the new estimator the statistical error was reduced by a factor of about 30 compared to the other two estimators. The results for the running coupling are summarized in table 4. For comparison I computed $\bar{g}^{2}$ to 1,2 and 3-loop order perturbation theory using the result for $\mathrm{L}=48$ as start value for the integration of eq. 28. The numerical results for $L=12,16,24$ and 32 are reproduced within two standard deviations. The larger deviations for $L=6$ and 8 are well described by corrections to scaling computed in perturbation theory [四]. 
Finally I also checked whether the new estimator also provides an improvement for the measurement of the bulk correlation length $\xi_{b u l k}$. Therefore I simulated the model at $\beta=1.5$. The correlation length is $\xi_{\text {bulk }}=11.09(2)$ [6]. Hence the result from a lattice of size $L=64$ should give $\xi_{\text {bulk }}$ to a good approximation. The effective correlation length for the three different estimators at the distances $\tau=10,20$ and 30 are given in table 5 . The cluster-improved estimator is superior to the new improved estimator but there is still an advantage for the new improved estimator compared to the conventional one by a factor of 1.4 at $\tau=10$ that grows to 2.8 at $\tau=30$ in the statistical error.

\subsection{The 2D $X Y$ model.}

I simulated the $2 \mathrm{D} X Y$ model at $\beta=0.92$ in the massive phase, at $\beta=1.1197$, which is the best estimate for the critical coupling [11] and at $\beta=1.2$ and 1.3 in the massless phase. All simulations consist of 25000 measurements. The results for the correlation length obtained from the different estimators are summarized in table 6 .

Let me again first discuss the reduction of the statistical error by the new improved estimator. At $\beta=1.3$ the statistical error of the new estimator is about 11 times smaller than that of the conventional one and about 9 times smaller than that of the cluster estimator. At $\beta=1.2$ the gain in the statistical accuracy is about a factor of 9 compared to the conventional estimator and about 6 compared to the cluster estimator for lattices larger than $L=8$. At $\beta_{c}$ the gain is reduced to a factor of 6 and 4 respectively. At $\beta=0.92$ the improvement depends very much on the lattice size. While for the smallest lattice size $L=8$ there is still an improvement of a factor of 2 compared to the cluster estimator, at $L=64$ the cluster estimator is clearly better by a factor of almost 4 . On the other hand the new estimator stays more accurate than the conventional estimator for all lattice sizes considered.

The dependence of the improvement on the distance $\tau$ is qualitatively the same as for the $O(3)$ model. Therefore I omit a detailed discussion here.

Now let us turn to the physical interpretation of the results. In the last column of table 6 I give the running coupling $\bar{g}^{2}$ computed from the best estimate for $\xi$. For $\beta=1.2$ and 1.3 the values stabilize for $L \geq 24$ within the small error bars. The small deviations for the smaller $L$ are consistent with corrections that die out with a negative power of $L$. This behaviour nicely reveals the scale invariance of a massless theory.

For $\beta=\beta_{c}$ there is a tiny increase of the coupling $\bar{g}^{2}$ over the whole range of $L$ and the value for $L=64$ is still far away from the predicted $L=\infty$ limit $\pi / 2$. 
However this behaviour is completely explained by

$$
\left.\bar{g}^{2}\right|_{\beta_{c}}=\frac{\pi}{2}-\frac{\pi}{4} \frac{g_{0}^{2}}{1+g_{0}^{2} \ln L}
$$

which can be derived from the KT-theory [10]. Results of fits according eq. (38) are given in table 7 . When lattices of size $L \leq 8$ are discarded the fits have an acceptable $\chi^{2} / d o f$ and $g_{0}^{2}$ remains stable when further data-points are discarded.

At $\beta=0.92 \bar{g}^{2}$ clearly increases with increasing lattice size $L$. In order to measure $\xi_{\text {bulk }}$ one simulates lattices with $L>>\xi_{b u l k}$. Here smaller distances than $\tau=L$ are sufficient to get a good estimate for $\xi_{b u l k}$ from $\xi_{\text {eff }}$. Therefore I evaluated $\xi_{\text {eff }}$ for $\beta=0.92$ and $L=64$ at various distances. The results are given in table 8 . At $\tau=10$ the statistical error of the cluster estimator is much smaller than that of the new improved estimator. But still the conventional one is worse by a factor of 1.3 .

\section{Conclusion}

I showed that the new improved estimator drastically reduces the statistical error of the correlation length of finite systems. At values of the running coupling $\bar{g}^{2}$ of the 2D $O(3)$ model, such as used by Lüscher et al. [4 the error was reduced by a factor of 4 up to 8 compared to the cluster-improved estimator. At $\bar{g}^{2} \approx 1 / 4$ a reduction of the error by a factor of 30 is reached.

On the other hand the error reduction seems to vanish in the limit $L / \xi_{\text {bulk }} \rightarrow \infty$. Nevertheless at $L \approx 6 \xi_{\text {bulk }}$, which might be sufficient for the determination of the bulk correlation length, there is still an improvement of a factor of 1.3 up to 1.4 compared to the conventional estimator, which might be interesting for models where no efficient cluster algorithm is known. A direct application of this method to gauge theories is not possible since there is no exact global symmetry that could be employed for the definition of an improved observable. A possible extension of the idea is an implementation of a multigrid scheme that has blocks with length one in time direction and $1,2,4 \ldots L$ in spatial direction.

As predicted, the running coupling $\bar{g}^{2}$ nicely signalled the presence of the massless phase of the 2D $X Y$ model. At fixed $\beta>\beta_{c}$ the coupling $\bar{g}^{2}$ rapidly approached a fixed point with increasing lattice size.

In contrast to that situation, at $\beta=5.0$ the $\bar{g}^{2}$ of the $O(3)$ model decreased with decreasing $L$. The change of $\bar{g}^{2}$ with doubling the lattice size was more than 100 times the statistical error. The change of $\bar{g}^{2}$ with varying length scale is almost perfectly described by the Callan-Symanzik $\beta$-function computed to three-loop order in perturbation theory. 


\section{Acknowledgement}

I would like to thank U. Wolff for many helpful discussions on the subject of this article. I thank K. Pinn and R. Sommer for a final discussion of the manuscript. 


\section{References}

[1] M.P. Nightingale, Physica A 83 (1976) 561.

[2] L. Onsager, Phys. Rev. 65 (1944) 117.

[3] M. Lüscher, Phys. Lett. B 118 (1982) 391.

[4] M. Lüscher, P. Weisz and U. Wolff, Nucl. Phys. B 359 (1991) 221.

[5] U. Wolff, Phys.Rev.Lett. 62 (1989) 361.

[6] U. Wolff, Nucl.Phys.B 334 (1990) 581.

[7] G. Parisi, Phys.Rept. 103 (1984) 203;

G. Parisi, R. Petronzio and F. Rapuano, Phys. Lett.B 128 (1983) 418.

[8] K. Symanzik, Cutoff dependence in lattice $\phi_{4}^{4}$ theory, in Recent developements in gauge theories (Cargèse 1979) ed. G. 't Hooft et al. (Plenum New York, 1980).

[9] M. Lüscher, Comput. Phys. Commun. 79 (1994) 100.

[10] See for example:

J.M. Kosterlitz and D.J. Thouless, J. Phys. C6, 1181 (1973);

J.M. Kosterlitz, J. Phys. C7, 1046 (1974);

S.T. Chui and J.D. Weeks, Phys. Rev. B14, 4978 (1976);

J.V. José, L.P. Kadanoff, S. Kirkpatrick, and D.R. Nelson, Phys. Rev. B16, 1217 (1977);

T. Ohta and K. Kawasaki, Prog. Theor. Phys. 60, 365 (1978);

D.J. Amit, Y.Y. Goldschmidt, and G. Grinstein, J. Phys. A13, 585 (1980).

[11] M. Hasenbusch, M. Marcu, K. Pinn, Physica A 208 (1994) 124.

[12] A.M. Polyakov, Phys. Lett. B 59 (1975) 79.

[13] U. Wolff, Nucl.Phys.B 322 (1989) 759. 


\section{Figure captions}

Figure 1: The relative error of the time-slice correlation function $\langle G(\tau)\rangle$ computed from the conventional, the cluster-improved and the new improved estimator as a function of the distance $\tau$ for $L=64$ and $\beta=1.8932$.

Figure 2: The relative error of the effective correlation length computed from the conventional, the cluster-improved and the new improved estimator of the correlation function as a function of the distance $\tau$ for $L=64$ and $\beta=1.8932$. 


\begin{tabular}{|c|c|r|c|c|c|c|}
\hline & $\beta$ & \multicolumn{1}{|c|}{$L$} & $\xi_{\text {con }}$ & $\xi_{\text {clu }}$ & $\xi_{\text {imp }}$ & $\bar{g}^{2}$ \\
\hline $\mathrm{x}$ & 1.9637 & 6 & $8.084(25)$ & $8.131(17)$ & $8.1295(25)$ & $0.7381(2)$ \\
$\mathrm{x}$ & 2.0100 & 8 & $10.867(33)$ & $10.835(29)$ & $10.8288(30)$ & $0.7388(2)$ \\
& 2.0809 & 12 & $16.339(58)$ & $16.350(34)$ & $16.2874(42)$ & $0.7368(2)$ \\
$\mathrm{x}$ & 2.1260 & 16 & $21.663(68)$ & $21.715(43)$ & $21.6645(50)$ & $0.7385(2)$ \\
& 2.2408 & 32 & $43.31(13)$ & $43.321(82)$ & $43.262(11)$ & $0.7397(2)$ \\
\hline & 1.9633 & 12 & $14.716(41)$ & $14.712(28)$ & $14.6969(42)$ & $0.8165(2)$ \\
& 2.0108 & 16 & $19.478(59)$ & $19.538(37)$ & $19.5873(51)$ & $0.8169(2)$ \\
& 2.0784 & 24 & $29.192(85)$ & $29.284(50)$ & $29.3862(75)$ & $0.8167(2)$ \\
& 2.1264 & 32 & $39.17(11)$ & $39.288(71)$ & $39.1900(92)$ & $0.8165(2)$ \\
& 2.2428 & 64 & $78.34(22)$ & $78.50(14)$ & $78.450(20)$ & $0.8158(2)$ \\
\hline $\mathrm{x}$ & 1.6050 & 6 & $5.658(18)$ & $5.665(9)$ & $5.6651(22)$ & $1.0591(4)$ \\
$\mathrm{x}$ & 1.6589 & 8 & $7.568(23)$ & $7.537(12)$ & $7.5571(30)$ & $1.0586(4)$ \\
$\mathrm{x}$ & 1.6982 & 10 & $9.433(29)$ & $9.422(14)$ & $9.4350(33)$ & $1.0599(4)$ \\
$\mathrm{x}$ & 1.7306 & 12 & $11.303(36)$ & $11.333(17)$ & $11.3247(41)$ & $1.0596(4)$ \\
$\mathrm{x}$ & 1.7800 & 16 & $15.075(40)$ & $15.107(23)$ & $15.1162(51)$ & $1.0585(4)$ \\
& 1.8460 & 24 & $22.597(66)$ & $22.636(35)$ & $22.6511(72)$ & $1.0596(4)$ \\
& 1.8931 & 32 & $30.041(91)$ & $30.206(49)$ & $30.1971(96)$ & $1.0597(3)$ \\
\hline & 1.6047 & 12 & $9.311(32)$ & $9.295(14)$ & $9.3040(43)$ & $1.2898(6)$ \\
& 1.6583 & 16 & $12.462(41)$ & $12.458(20)$ & $12.4548(59)$ & $1.2846(6)$ \\
& 1.6985 & 20 & $15.627(64)$ & $15.648(23)$ & $15.6274(64)$ & $1.2798(5)$ \\
& 1.7306 & 24 & $18.760(60)$ & $18.849(28)$ & $18.8174(77)$ & $1.2754(5)$ \\
& 1.7793 & 32 & $25.148(82)$ & $25.193(33)$ & $25.176(11)$ & $1.2711(6)$ \\
& 1.8460 & 48 & $37.96(11)$ & $37.927(49)$ & $37.868(16)$ & $1.2676(5)$ \\
& 1.8932 & 64 & $50.75(15)$ & $50.694(70)$ & $50.584(22)$ & $1.2652(6)$ \\
\hline
\end{tabular}

Table 1: Results for the correlation length $\xi$ of the 2D $O(3)$ model obtained from various estimators of the correlation function. The subscripts con, clu and imp denote the conventional, the cluster-improved and the new improved estimator respectively. In addition I give the result for the running coupling $\bar{g}^{2}$ obtained from the most accurate result for $\xi$. The simulations that have a direct counter-part in table 2 of ref. 4 are marked by an $\mathrm{x}$. 


\begin{tabular}{|c|c|c|}
\hline$L / a$ & $\delta_{1}$ & $\delta_{2}$ \\
\hline 6 & -0.00657 & 0.00289 \\
8 & -0.00364 & 0.00176 \\
12 & -0.00160 & 0.00085 \\
16 & -0.00090 & 0.00050 \\
\hline
\end{tabular}

Table 2: Perturbative results for the finite lattice spacing artefacts taken from ref. [4].

\begin{tabular}{|c|l|c|l|c|}
\hline $\operatorname{disc}$ & $\sigma(2,1.0595)$ & $c_{2}$ & $c_{4}$ & $\chi^{2} /$ dof \\
\hline 0 & $1.2641(6)$ & $1.89(13)$ & $-34.7(3.0)$ & 0.38 \\
1 & $1.2638(8)$ & $2.03(24)$ & $-44 .(14)$. & 0.34 \\
2 & $1.2639(10)$ & $1.95(47)$ & $-37 .(42)$. & 0.49 \\
\hline
\end{tabular}

Table 3: Results of fits for $\Sigma(2,1.0595, a / L)$ according to eq. 38. disc gives the number of discarded data points with small $L$.

\begin{tabular}{|r|c|c|c|c|c|}
\hline$L$ & $\bar{g}^{2}$ & 1-loop & 2-loop & 3-loop & $\bar{g}^{2} \delta\left(\bar{g}^{2}, a\right)$ \\
\hline 6 & $0.22399(4)$ & 0.22435 & 0.22373 & 0.22369 & -0.00030 \\
8 & $0.22624(4)$ & 0.22667 & 0.22613 & 0.22609 & -0.00017 \\
12 & $0.22959(4)$ & 0.23004 & 0.22960 & 0.22957 & -0.00007 \\
16 & $0.23210(3)$ & 0.23249 & 0.23213 & 0.23211 & -0.00004 \\
24 & $0.23570(3)$ & 0.23603 & 0.23580 & 0.23579 & \\
32 & $0.23836(3)$ & 0.23861 & 0.23847 & 0.23846 & \\
48 & $0.24234(3)$ & & & & \\
\hline
\end{tabular}

Table 4: Results for the running coupling $\bar{g}^{2}$ at $\beta=5.0$ on lattices of the size $L=6$ up to $48 \cdot \bar{g}^{2}$ is computed form the new improved estimator of the correlation function. The Monte Carlo result is compared with the result of the Callan-Symanzik $\beta$-function to 1, 2 and 3-loop order. As starting point of the integration I took the MC result for $L=48$. In the last column I give the finite lattice spacing artefact for a step with scaling factor 2 as computed from perturbation theory. 


\begin{tabular}{|c|l|c|c|}
\hline$t$ & \multicolumn{1}{|c|}{$\xi_{\text {con }}$} & $\xi_{\text {clu }}$ & $\xi_{\text {imp }}$ \\
\hline 11 & $11.023(33)$ & $11.031(15)$ & $11.031(24)$ \\
22 & $10.923(85)$ & $11.022(16)$ & $11.007(48)$ \\
33 & $10.70(26)$ & $11.007(21)$ & $11.034(94)$ \\
\hline
\end{tabular}

Table 5: Results for the effective correlation length for various distances $t$ at $L=64$ at $\beta=1.5$. The results can be compared with $\xi=11.09(2)$ obtained in ref. [6] on a $128^{2}$ lattice. 


\begin{tabular}{|c|r|c|c|c|c|}
\hline$\beta$ & $L$ & $\xi_{\text {con }}$ & $\xi_{\text {clu }}$ & $\xi_{\text {imp }}$ & $\bar{g}^{2}$ \\
\hline 1.3 & 4 & $7.49(5)$ & $7.46(4)$ & $7.476(6)$ & $1.0701(9)$ \\
1.3 & 6 & $11.49(7)$ & $11.44(5)$ & $11.383(8)$ & $1.0542(7)$ \\
1.3 & 8 & $15.26(10)$ & $15.18(7)$ & $15.265(10)$ & $1.0481(7)$ \\
1.3 & 12 & $22.97(16)$ & $22.93(12)$ & $22.952(15)$ & $1.0457(7)$ \\
1.3 & 16 & $30.65(21)$ & $30.85(16)$ & $30.661(18)$ & $1.0437(6)$ \\
1.3 & 24 & $46.20(31)$ & $46.15(23)$ & $46.083(26)$ & $1.0416(6)$ \\
1.3 & 32 & $61.25(39)$ & $61.18(28)$ & $61.420(35)$ & $1.0420(6)$ \\
1.3 & 48 & $92.25(65)$ & $91.80(50)$ & $92.192(49)$ & $1.0413(6)$ \\
\hline 1.2 & 4 & $6.53(4)$ & $6.54(3)$ & $6.502(7)$ & $1.2304(13)$ \\
1.2 & 6 & $9.74(6)$ & $9.75(4)$ & $9.856(9)$ & $1.2175(11)$ \\
1.2 & 8 & $13.19(9)$ & $13.19(6)$ & $13.189(12)$ & $1.2131(11)$ \\
1.2 & 12 & $20.05(14)$ & $19.96(10)$ & $19.839(16)$ & $1.2097(10)$ \\
1.2 & 16 & $26.43(17)$ & $26.56(12)$ & $26.419(22)$ & $1.2112(10)$ \\
1.2 & 24 & $40.16(27)$ & $39.59(18)$ & $39.748(28)$ & $1.2076(9)$ \\
1.2 & 32 & $53.09(31)$ & $53.07(22)$ & $52.975(34)$ & $1.2081(8)$ \\
1.2 & 48 & $79.55(52)$ & $79.59(40)$ & $79.468(52)$ & $1.2080(8)$ \\
\hline 1.1197 & 4 & $5.75(4)$ & $5.74(3)$ & $5.695(7)$ & $1.4047(17)$ \\
1.1197 & 6 & $8.57(6)$ & $8.57(3)$ & $8.554(10)$ & $1.4029(16)$ \\
1.1197 & 8 & $11.39(7)$ & $11.33(5)$ & $11.333(13)$ & $1.4118(16)$ \\
1.1197 & 12 & $16.76(10)$ & $16.87(7)$ & $16.919(19)$ & $1.4185(16)$ \\
1.1197 & 16 & $22.45(15)$ & $22.52(9)$ & $22.446(23)$ & $1.4256(15)$ \\
1.1197 & 24 & $33.49(23)$ & $33.38(14)$ & $33.390(36)$ & $1.4376(15)$ \\
1.1197 & 32 & $44.26(29)$ & $44.41(18)$ & $44.369(42)$ & $1.4424(14)$ \\
1.1197 & 48 & $66.33(44)$ & $65.92(30)$ & $66.082(61)$ & $1.4527(13)$ \\
1.1197 & 64 & $88.59(59)$ & $87.39(35)$ & $87.898(89)$ & $1.4562(15)$ \\
$\beta_{c}$ & $\infty$ & & & & 1.570796 \\
\hline 0.92 & 4 & $3.73(3)$ & $3.706(12)$ & $3.688(6)$ & $2.169(4)$ \\
0.92 & 8 & $6.00(6)$ & $6.044(18)$ & $6.018(11)$ & $2.659(3)$ \\
0.92 & 16 & $8.46(9)$ & $8.578(25)$ & $8.630(25)$ & $3.708(11)$ \\
0.92 & 32 & $10.78(28)$ & $10.193(26)$ & $10.221(86)$ & $6.279(16)$ \\
0.92 & 64 & & $10.559(42)$ & $8.8(1.2)$ & $12.12(5)$ \\
\hline
\end{tabular}

Table 6: Results for the correlation length $\xi$ of the 2D $X Y$ model obtained from different estimators of the correlation function. The subscripts con, clu and imp denote the conventional, the cluster-improved and the new improved estimator respectively. In addition I give the result for the running coupling $\bar{g}^{2}$ obtained from the most accurate result for $\xi$. 


\begin{tabular}{|c|c|c|c|c|c|}
\hline disc & 0 & 1 & 2 & 3 & 4 \\
$g_{0}^{2}$ & $0.3466(4)$ & $0.3618(4)$ & $0.367(5)$ & $0.372(6)$ & $0.372(7)$ \\
$\chi^{2} /$ dof & 8.4 & 1.4 & 0.9 & 0.2 & 0.3 \\
\hline
\end{tabular}

Table 7: Results for fits of the running coupling $\bar{g}^{2}$ of the $2 \mathrm{D} X Y$ model at $\beta_{c}$ following eq. 39. disc gives the number of data-points with small $L$ that are discarded.

\begin{tabular}{|c|l|l|l|}
\hline$\tau$ & \multicolumn{1}{|c|}{$\xi_{\text {con }}$} & \multicolumn{1}{c|}{$\xi_{\text {clu }}$} & \multicolumn{1}{|c|}{$\xi_{\text {imp }}$} \\
\hline 10 & $10.568(24)$ & $10.552(8)$ & $10.568(18)$ \\
20 & $10.56(6)$ & $10.556(11)$ & $10.53(4)$ \\
30 & $10.66(17)$ & $10.537(14)$ & $10.55(9)$ \\
\hline
\end{tabular}

Table 8: Results for the correlation length of the 2D $X Y$ model at $\beta=0.92$ and $L=64$. The effective correlation length is computed from the conventional con, the new improved $\mathrm{imp}$ and the cluster-improved clu estimator at the distances $\tau=10,20$ and 30. The results can be compared with $\xi=10.69(8)$ obtained form a $128^{2}$ lattice [13]. 
This figure "fig1-1.png" is available in "png" format from: http://arxiv.org/ps/hep-lat/9408019v1 
This figure "fig1-2.png" is available in "png" format from: http://arxiv.org/ps/hep-lat/9408019v1 